\title{
Automatic spatial coding of perceived gaze direction is revealed by the Simon effect
}

\author{
MARCO ZORZI, DANIELA MAPELLI, ELENA RUSCONI, and CARLO UMILTÀ \\ University of Padua, Padua, Italy
}

\begin{abstract}
In a typical Simon task, the (irrelevant) spatial position of the stimulus interferes with the processing of the salient characteristic(e.g., color). We used the Simon effect to investigate the automatic processing of gaze cues. We show that a simple drawing of schematic eyes automatically generates a spatially defined code of gaze direction. Although completely irrelevant to the task, direction of gaze influenced reaction times in a spatially selective two-choice discrimination based on eye color. Moreover, in one experiment employing an orthogonal manipulation of stimulus position and gaze direction, we found that coding of gaze direction is independent of stimulus spatial coding. Our finding of a "gazedirection Simon effect" is congruent with the hypothesis that gaze direction is coded by a specialized mechanism.
\end{abstract}

What makes eyes special? Common experience suggests that the eyes are a point of attraction when we look at faces. Even infants look at the eyes more than anywhere else in the face (Maurer, 1985). Notably, a simple stimulus made with three blobs corresponding to eyes and mouth within a frame is sufficiently face-like to induce newborns to look preferentially to it (Valenza, Simion, Macchi Cassia, \& Umiltà, 1996); moreover, newborns show a preference for a face with the eyes open in comparison with the same face with the eyes closed (Batki, Baron-Cohen, Wheelwright, Connellan, \& Ahluwalia, 2000).

In many cases, the eyes are an important source of social information for the observer (Argyle \& Cook, 1976). Gaze direction may indicate the spatial position of an interesting event in the environment (i.e., food or danger) and may therefore activate a form of shared attention between two conspecifics (Butterworth \& Jarrett, 1991). Baron-Cohen (1994) proposed the existence of an innate brain mechanism subserving processing of gaze direction (the eye direction detector [EDD]). Recent studies have shown that babies can detect gaze direction very early in life (Maurer, 1985; Vecera \& Johnson, 1995) and use it to direct attention (Hood, Willen, \& Driver, 1998).

A number of studies suggest that social cues such as direction of gaze, but also head, mouth, and hand movements, are processed by a specific cortical region in and near the superior temporal sulcus (STS; see Allison, Puce, \& McCarthy, 2000, for a review). STS neurons

This work was supported by a McDonnell-Pew Cognitive Neuroscience Program grant to M.Z. and C.U. and by grants from the Consiglio Nazionale delle Ricerche and the Ministero della Università e della Ricerca Scientifica e Tecnologica to C. U. We thank Jari Hietanen, Steve Langton, and an anonymous reviewer for helpful comments on an earlier version of the manuscript. Part of this work was presented at the annual meeting of the Cognitive Neuroscience Society, San Francisco, April 2000. Correspondence concerning this article should be addressed to C. Umiltà, Dipartimento di Psicologia Generale, Via Venezia 8, 35131 Padova, Italy (e-mail: carlo.umilta@unipd.it). recorded in monkeys respond selectively to faces and to different directions of gaze (Bruce, Desimone, \& Gross, 1981; Perrett et al., 1985), and STS activity has been related to perception of gaze in recent neuroimaging studies with human participants (Hoffman \& Haxby, 2000; Wicker, Michel, Henaff, \& Decety, 1998).

There are two lines of evidence that suggest that gaze direction is processed rapidly and automatically (see, e.g., Langton, Watt, \& Bruce, 2000, for a review). One makes use of a version of Posner's (1980) cuing paradigm. Basically, it shows that central gaze cues trigger reflexive visual orienting the way abrupt, peripheral visual cues are known to do, and perhaps as directional symbolic cues do also (Hommel, Pratt, Colzato, \& Godijn, 2001; Tipples, 2002). For example, Driver, Davis, Kidd, Maxwell, Ricciardelli, and Baron-Cohen (1999; see also Friesen \& Kingstone, 1998) presented a picture of a face gazing in a particular direction to cue observers to the position of a target stimulus. It was found that the observers could not avoid attending to the location signaled by gaze direction even when they knew that the cue was invalid and/or were explicitly instructed to ignore it. Also, the role of gaze direction in spatial attention was suggested by the imaging study of Hoffman and Haxby (2000), who showed that faces with averted eye gaze elicited activity in the intraparietal sulcus, which is known to be involved in spatial attention (see, e.g., Corbetta, Shulman, Miezin, \& Peterson, 1995). Direction of gaze very likely needs to be transformed into a directional, spatial code to drive a shift of attention. The demonstration that gaze cues automatically trigger orienting strongly suggests that such a transformation takes place.

Other evidence of transformation of gaze direction into a spatial code derives from a second line of evidence that makes use of a Stroop-like interference paradigm. For example, in one experiment Langton (2000) used a combination of head and gaze cues. Participants were asked to respond to the orientation of the head (i.e., the task-relevant 
information) and to ignore gaze direction (i.e., the taskirrelevant but potentially interfering information). The results showed that speed of response depended on whether or not gaze direction was congruent with head direction. Langton obtained similar results in another experiment, in which the task-relevant information was a spoken directional word and the task-irrelevant information was, again, gaze direction. This is no doubt strong evidence of the extraction of spatial information from gaze. However, although the participants were instructed to ignore the direction of gaze, the task they were asked to perform still required processing of spatial information carried by the target. In our view, even more compelling evidence in support of the automatic generation of a spatial code concerning gaze direction would come from an experimental condition in which the task required processing of a nonspatial feature of the stimulus.

In the present study, we used a classic and robust behavioral paradigm called the Simon task (Lu \& Proctor, 1995; Simon \& Craft, 1970; Umiltà \& Nicoletti, 1990), in which spatial information is completely irrelevant to the task. In a typical Simon task, participants are presented with one of two colored lights and are instructed to press the left key in response to one color and the right key in response to the other. Even though stimulus position is irrelevant for performing the task (i.e., the color discrimination), the response is faster, and often more accurate, when the position of the stimulus (left or right on the display) corresponds to the position (left or right) of the response key (i.e., corresponding $\mathrm{S}-\mathrm{R}$ pairings) than when it does not (i.e., noncorresponding S-R pairings). It is clear that in the Simon task a spatial code is generated for the irrelevant stimulus-locational attribute, which, in turn, affects response selection (see, e.g., Lu \& Proctor, 1995; Zorzi \& Umiltà, 1995).

In the variant of the Simon task used in the present study, the task-relevant information was eye color, whereas the task-irrelevant information was gaze direction. Experiment 1 was devoted to showing that there is a Simon effect solely attributable to gaze direction, and in Experiments 2 and 3 we assessed whether the effect is specific for eye-like stimuli. The use of the Simon task allows one to investigate whether the spatial code for gaze direction is computed by a mechanism different from a generalpurpose spatial coding mechanism. We varied orthogonally the location of the gaze stimulus in the visual field and the gaze direction, and asked whether the Simon effect attributable to spatial position and the Simon effect attributable to gaze direction are additive or interactive. If they are additive, by applying the logic of the additive factor method (AFM; Sternberg, 1969), one may conclude that the two spatial codes are produced by two independent mechanisms. This was done in Experiment 4.

\section{EXPERIMENT 1}

On each trial, the participants were presented with an eye-like stimulus (see Figure 1) consisting of two ellip- tic frames, each of which contained a colored circle ("iris"). The stimulus appeared in the center of the computer screen, and the participants' task was to respond quickly and accurately to the color of the "irises" (blue or green) by pressing one of two response keys located left and right of body midline. The participants were not informed that the stimulus was intended to resemble eyes, and the instructions referred only to the shapes presented in the display. The colored circles could be located in the center, on the left, or on the right within the elliptic frames, to produce three directions of gaze. It was still possible, however, that the position of the "iris" within the frame could generate a spatial position code. This was controlled by centering either the frames (frame-centered condition) or the colored circles (targetcentered condition) with respect to the fixation point.

We used a simple drawing of schematic eyes, as Kingstone, Friesen, and Gazzaniga (2000) did. Other studies on gaze direction have instead used schematic faces (Friesen \& Kingstone, 1998) or pictures of real faces (Driver et al., 1999; Hietanen, 1999; Langton, 2000).

\section{Method}

Eighteen students of the University of Padua (mean age $=$ 24 years) participated in the experiment. All of them had normal or corrected-to-norm al vision. The participants were seated about $60 \mathrm{~cm}$ from the screen of a 14-in. Trinitron color monitor driven by a Power Macintosh 6100/66 computer. The visual display comprised a $0.7^{\circ} \times 0.7^{\circ}$ central fixation cross. The overall size of the stimulus, which comprised two elliptic frames ( $8.6^{\circ}$ in size), was $20^{\circ}$. The size of the colored target was $3.8^{\circ}$, and the stimulus was centrally presented.

There were two conditions of alignment of the eyes with respect to the fixation point. In the frame-centered condition, the frames were always in the same display position for all the stimuli, and the colored circle was located in the center, left, or right within each frame. In the target-centered condition, the colored circles were always in the same position on the screen, and the frames were moved to the left or to the right to create the different gaze directions. Note that this produced a slight shift of the entire configuration to the side,

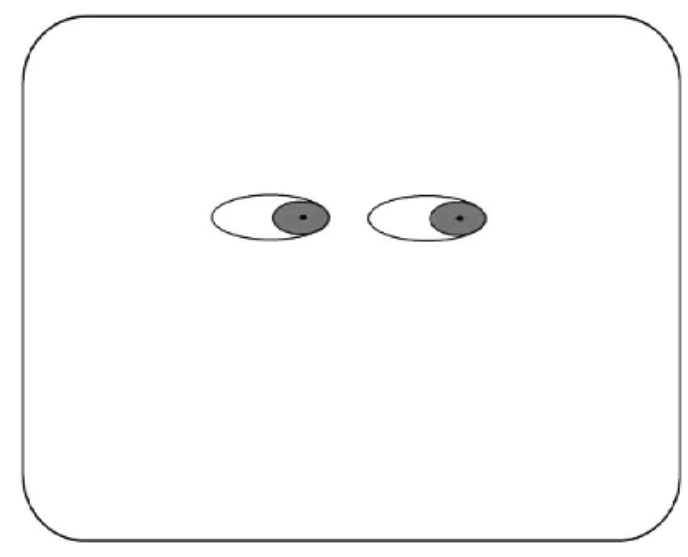

Figure 1. Example of the display used in Experiment 1: Green "irises" with right gaze direction. The entire set of stimuli was made by the combination of two colors (blue and green) and three different gaze directions (center, right, and left). 
but in the direction opposite gaze direction (e.g., the left-gazing stimulus was slightly shifted to the right with respect to the centergazing stimulus).

The responses were executed by pressing one of two keys on the computer keyboard (D and L). The D key was located to the left of body midline and was operated by the left index finger, whereas the L key was located to the right of body midline and was operated by the right index finger.

The experiment was divided into two different blocks, with each block comprising 180 trials in one alignment condition (frame centered vs. target centered). The order of the alignment conditions was counterbalanced between participants. An equal number of pictures was presented, in random order, for the left, right, and central gaze directions. Each participant started the session with 24 practice trials. A trial started with the presentation of a central fixation cross for $450 \mathrm{msec}$. After $50 \mathrm{msec}$ from fixation offset, a stimulus was presented until a keypress response was made. Half of the participants were instructed to respond to the blue color by pressing the right key (L) and to the green color by pressing the left key (D), whereas the other half received the opposite instructions. The blue and green stimulus colors were evenly distributed among the three gaze-direction conditions.

\section{Results and Discussion}

Errors were few (less than 4\%) and evenly distributed among conditions. Therefore, they were not analyzed. An analysis of variance (ANOVA) was performed on correct mean reaction times (RTs) calculated for each subject as a function of alignment condition (target centered vs. frame centered) and spatial correspondence between gaze direction and response side (corresponding vs. noncorresponding vs. neutral). Even though gaze direction was irrelevant to the task, the participants were faster to respond to the color when the side of the response key corresponded with gaze direction than when it did $\operatorname{not}\left[F(2,34)=12.32, M S_{\mathrm{e}}=432.7, p<.001\right]$. RTs for corresponding trials were significantly shorter than RTs for neutral (central) trials [380 vs. $390 \mathrm{msec} ; F(1,17)=$ $15.00, p<.01]$, which, in turn, were significantly shorter than RTs for noncorresponding trials [390 vs. $404 \mathrm{msec}$; $F(1,17)=7.89, p<.025]$. The two alignment conditions (frame centered vs. target centered) were not statistically different $(F<1)$, and the alignment factor did not interact with spatial correspondence. Note that the 24-msec effect (i.e., the RT difference between noncorresponding trials and corresponding trials) is not a regular Simon effect, because the stimulus was not lateralized. Apparently, this was a "gaze-direction Simon effect."

After completion of the experiment, the participants were asked to describe the stimuli they had just seen. All but 1 (17 of 18) spontaneously referred to them as eyes.

\section{EXPERIMENT 2}

To confirm that the directional effect was indeed produced by the eye-likeness of the stimuli, we ran a control experiment in which the stimuli had similar physical characteristics but were not eye-like (Figure 2). The participants were asked to respond to the color of the filled squares that were located in the center, on the left, or on the right within the larger square frames.

\section{Method}

Eighteen students of the University of Padua (mean age $=22$ ) who fulfilled the same criteria as did those in Experiment 1 participated in Experiment 2. The apparatus and procedure were as in Experiment 1 , with the exception that the two frames were squared $\left(8.6^{\circ}\right.$ in size $)$ and the colored target was a filled square $\left(2^{\circ}\right.$ in size). The size of the filled squares was smaller than that of the "irises" in Experiment 1, because we wanted to render the stimuli as little like eyes as possible. Because of that, in order to maintain the same eccentricity as in Experiment 1, the filled squares did not reach the border of the frame. As in Experiment 1, there were two conditions of stimulus alignment (frame centered vs. target centered) with respect to fixation.

\section{Results and Discussion}

Errors constituted fewer than 5\% of responses and were evenly distributed among conditions. They were not analyzed. None of the sources was significant in the ANOVA, which was based on the same factors as in Experiment 1. The main effect of spatial correspondence was very close to significance $\left[F(2,34)=3.25, M S_{\mathrm{e}}=694.0, p=.0519\right]$. Importantly, however, that was due entirely to the difference between neutral and noncorresponding trials [422 vs. $437 \mathrm{msec} ; F(1,17)=7.99, p<.025$ ], whereas RTs for corresponding and noncorresponding trials were very similar [432 vs. $437 \mathrm{msec} ; F(1,17)=1.46, p=.243$ ] . Thus, RT was not influenced by whether or not the spatial position of the colored square corresponded to the response side. Neither the factor of alignment nor its interaction with spatial correspondence was significant (both $F_{\mathrm{S}}<1.5$ ).

As in Experiment 1, after completion of the experiment, the participants were asked to describe the stimuli. None of them referred to them as eyes. To confirm that the eye-like stimuli were processed in a different way, a combined analysis of Experiments 1 and 2 was performed, with experiment (Experiment 1 vs. Experiment 2) as a between-subjects factor and correspondence (corre-

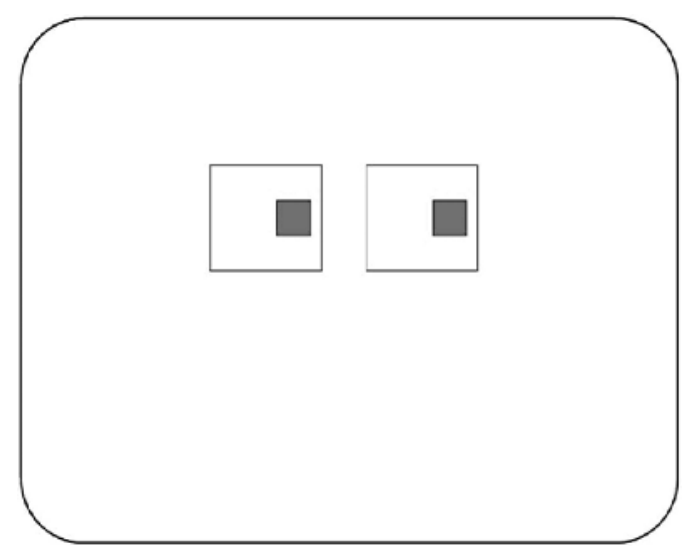

Figure 2. Example of the display used in Experiment 2: Green squares on the right side of squared frames. The entire set of stimuli was made by the combination of two colors (blue and green) and three different positions of the colored squares (center, right, and left). 
sponding vs. noncorresponding vs. neutral) as a withinsubjects factor. Mean RTs (collapsed over the two frametarget alignment conditions) are plotted in Figure 3. The ANOVA revealed a significant interaction between experiment and correspondence $\left[F(2,68)=4.11, M S_{\mathrm{e}}=\right.$ $281.1, p<.025]$. This shows that the effect we found in Experiment 1 was indeed a "gaze-direction Simon effect."

\section{EXPERIMENT 3}

The claim that the gaze-direction Simon effect is produced by the eye-likeness of the stimuli in Experiment 1 rests on the null result (i.e., the absence of a significant Simon effect) obtained with the control stimuli in Experiment 2. The purpose of Experiment 3 was to replicate these results using a more powerful, within-subjects design. Thus, the participants responded in two separate blocks to eye-like stimuli and to control stimuli.

\section{Method}

Twenty students of the University of Padua (mean age $=26.7$ ) who fulfilled the same criteria as did those in Experiment 1 participated in Experiment 3. None of them had taken part in either of the previous experiments. The apparatus and procedure were as in Experiment 1 . The stimuli used in Experiment 1 (eye-like) and Experiment 2 (control) were presented in two separate blocks. The order of the two blocks was fixed, with the control stimuli always presented before the eye-like stimuli. The procedure was specifically designed this way to avoid a carry-over effect of the eye-like stimuli on the control stimuli. That is, seeing the eye-like stimuli first would produce a strong tendency to perceive the squares of Experiment 2 as eyes. Indeed, one anonymous reviewer of this article pointed out that the control stimuli looked somewhat eye-like to him. However, when seen out of context (i.e., without any reference to eyes), the control stimuli were simply described as squares: None of the the participants in Experiment 2 described the stimuli as eyes or made reference to eyes (in sharp contrast to the participants in Experiment 1, all but 1 of whom spontaneously described the elliptical stimuli as eyes).

\section{Results and Discussion}

Errors constituted fewer than $2 \%$ of responses and were evenly distributed among conditions. They were not analyzed. An ANOVA was performed on correct RTs, calculated for each subject as a function of stimulus type (eyes vs. control), alignment condition (target centered vs. frame centered), and spatial correspondence (corresponding vs. noncorresponding vs. neutral).

The main effect of spatial correspondence was significant $\left[F(2,38)=8.12, M S_{\mathrm{e}}=321.8, p<.005\right]$. However, it was qualified by a significant interaction with stimulus type $\left[F(2,38)=3.33, M S_{\mathrm{e}}=265.2, p<.05\right]$. That is, the participants showed a spatial correspondence (Simon) effect only with the eye-like stimuli ( $18 \mathrm{msec}$ vs. $4 \mathrm{msec}$ ). For the control stimuli, RTs for corresponding $(410 \mathrm{msec})$, neutral (412 msec), and noncorresponding (414 msec) trials were not significantly different (for all contrasts, $p s>.1$ ). Although the difference only approached significance, for eye-like stimuli, RTs for corresponding trials were shorter than RTs for neutral (central) trials [391 vs. $398 \mathrm{msec} ; F(1,19)=4.01, p=.06$ ]. In turn, RTs for neutral trials were significantly shorter than RTs for noncorresponding trials [398 vs. $409 \mathrm{msec} ; F(1,19)=$ $4.55, p<.05]$. No other main effect or interaction was significant. Thus, the results mirror those of Experiments 1

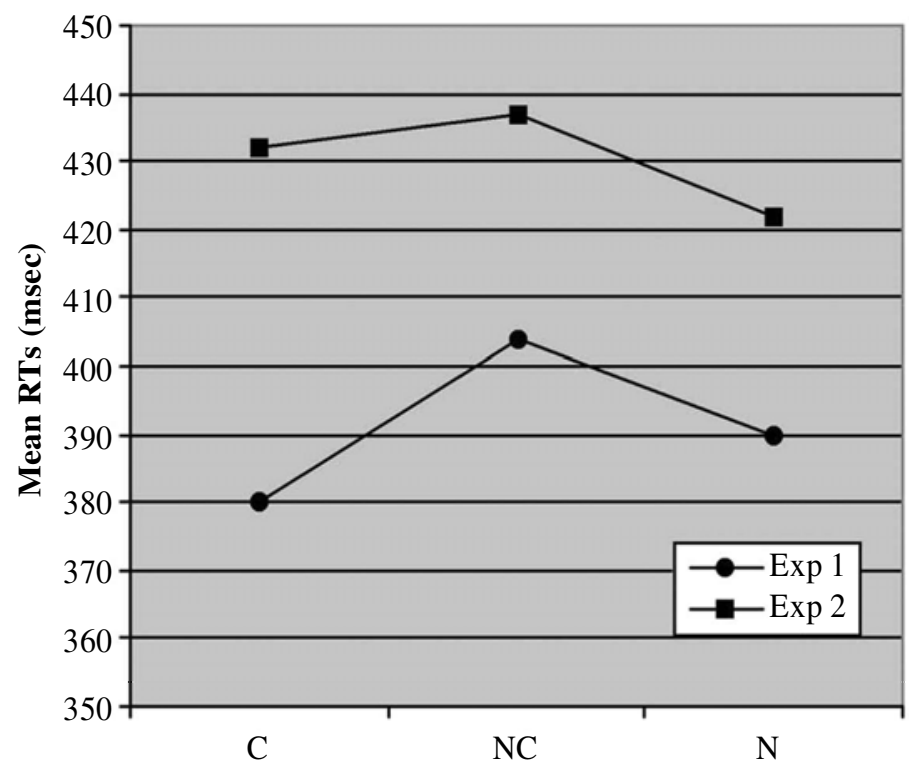

Figure 3. Mean reaction times (RTs) from Experiment 1 and Experiment 2 plotted as a function of correspondence and experiment. $\mathrm{C}=$ corresponding; $\mathrm{NC}=$ noncorresponding; $\mathbf{N}=$ neutral. 


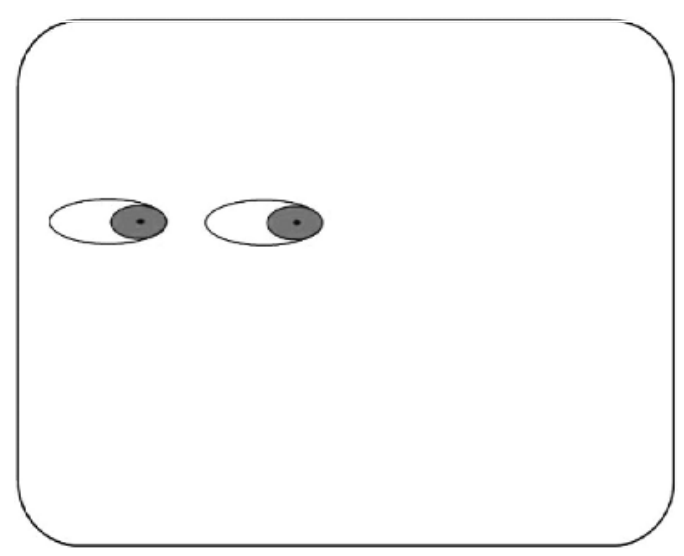

Figure 4. Example of the display used in Experiment 4: Green "irises" with right gaze direction positioned on the left side of the display. The entire set of stimuli was made by the combination of two colors (blue and green), three different gaze directions (center, right, and left), and two positions on the display (left and right).

and 2. Only the eye-like stimuli produced a significant effect of spatial correspondence - that is, a gaze-direction Simon effect. Here and in the previous experiments, the small, nonsignificant Simon effect for the control stimuli might be due to the slight bias of the square away from the edge of the square, which was lacking in the case of the eye-like stimuli.

In Experiment 3, the control stimuli were always presented before the eye-like stimuli. Even though there is no evidence whatsoever in the literature that the Simon effect emerges only with practice, there is the possibility that the difference between conditions in the present experiment were due to the fixed order of stimulus presentation. One can explore this possible confound by examining the data from Experiment 1 separately for the first and second blocks. The Simon effect was nearly identical in both blocks ( 24 and $25 \mathrm{msec}$, respectively).

\section{EXPERIMENT 4}

In Experiment 4, the participants were presented with the same eye-like stimuli as in Experiment 1 (with targetcentered alignment), but the stimulus could appear either on the left side or on the right side of the computer screen (Figure 4). The participants were asked to make a twochoice decision on the basis of the color of the "irises" and to ignore their spatial position. However, we expected the lateralized presentation of the stimulus to produce a regular Simon effect- that is, faster responses when the position of the stimulus corresponded to the position of the response key than when it did not. Therefore, we asked whether the effect of gaze direction could still be found and, if so, whether it would interact with the regular Simon effect or not. Evidence for non-interactivity (i.e., additivity) of the two effects would provide evidence for their reliance on different mechanisms (i.e., the AFM logic; Sternberg, 1969).

\section{Method}

Sixteen students of the University of Padua (mean age $=25$ ) who fulfilled the same criteria as did those in Experiment 1 participated in Experiment 4. The apparatus and procedure were as in Experiment 1 , with the exception that the eye-like stimulus was presented on the left or on the right of the display, so that the "iris" nearest the center was at a distance of $5^{\circ}$ from the fixation cross. For each display position, the alignment of the stimulus with respect to the fixation point was target centered. There were 120 trials for each of the two possible screen locations (left vs. right), for a total of 240 trials.

\section{Results and Discussion}

Errors constituted fewer than $3 \%$ of responses and were evenly distributed among conditions. They were not analyzed. Mean RTs were computed for each participant as a function of the correspondence between stimulus position and response side and of that between gaze direction and response side (see Figure 5). The ANOVA revealed that the participants were faster to respond when stimulus position and response side corresponded than when they did not [423 vs. $444 \mathrm{msec}$, respectively; $\left.F(1,15)=20.13, M S_{\mathrm{e}}=495.9, p<.001\right]$. This is a regular Simon effect, produced by the automatic coding of the (irrelevant) information about stimulus position ( $\mathrm{Lu}$ \& Proctor, 1995; Zorzi \& Umiltà, 1995). However, the participants were also faster to respond when gaze direction corresponded to the response side than when it did $\operatorname{not}\left[F(2,30)=15.40, M S_{\mathrm{e}}=491.3, p<.001\right]$. RTs were $420 \mathrm{msec}$ for corresponding gaze direction, $430 \mathrm{msec}$ for neutral (central) gaze direction, and $450 \mathrm{msec}$ for noncorresponding gaze direction. The differences between corresponding trials and neutral trials $[F(1,15)=7.86$, $p<.025]$ and between neutral trials and noncorresponding trials $[F(1,15)=12.02, p<.005]$ were significant. Crucially, the two effects did not interact $(F<1)$, suggesting that the effect of spatial correspondence and the effect of gaze direction are additive.

After completion of the experiment, the participants were asked to describe the stimuli. All of them referred to them as eyes.

\section{GENERAL DISCUSSION}

In this article, two basic claims are made. One is that gaze direction produces an automatic spatial code that, in turn, produces a Simon effect. The second is that the automatic spatial code is specific to eye-like stimuli and, thus, supports Baron-Cohen's (1994) EDD hypothesis. We believe that the present results strongly support the first claim and are compatible with the second. In particular, the results of Experiment 4 speak to the issue of the existence of a specialized spatial coding mechanism and provide evidence that the eye-like stimulus generates a spatially defined code of gaze direction that is independent of spatial coding of stimulus position. Recent accounts of the Simon effect (Lu \& Proctor, 1995; Tagliabue, Zorzi, Umiltà, \& Bassignani, 2000; Zorzi \& Umiltà, 1995) emphasize that coding of the (task-irrelevant) spatial information must be fast and automatic to influence a simple two-choice task based on nonspatial information. Simi- 


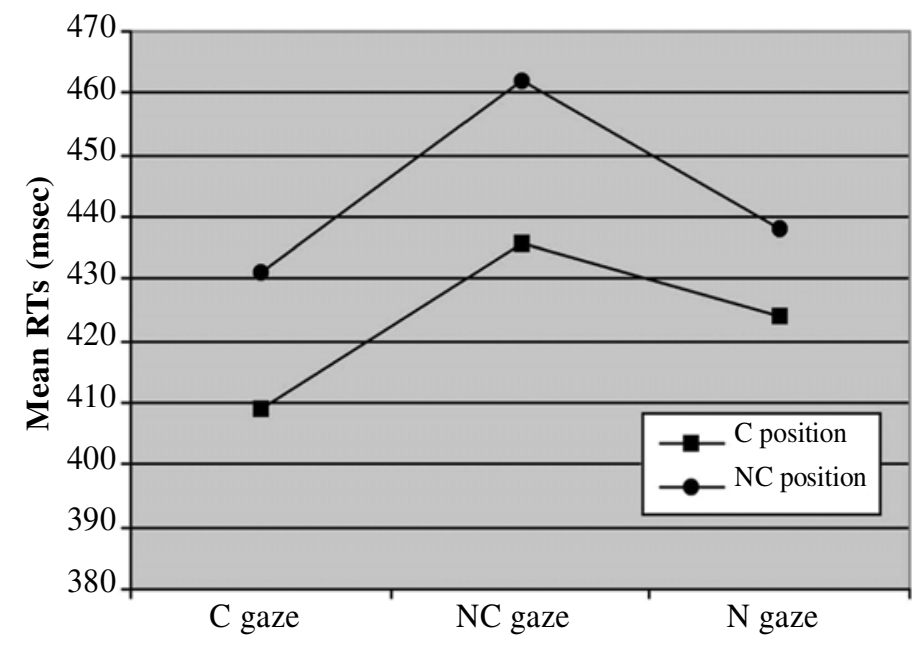

Figure 5. Mean reaction times (RTs) from Experiment 4 plotted as a function of correspondence between stimulus position and response side and between gaze direction and response side $(\mathrm{C}=$ corresponding; $\mathrm{NC}=$ noncorresponding; $\mathbf{N}$ = neutral).

larly, we must assume that gaze direction is fast and automatically detected at the initial stages of visual processing in order to influence RT in the color discrimination task.

Our study suggests that gaze direction is processed by a specialized mechanism, such as Baron-Cohen's (1994) EDD module. It is conceivable, however, that this mechanism operates not only on gaze but also on other social cues, such as head direction (see, e.g., Langton, 2000) and hand gestures (see, e.g., Langton \& Bruce, 2000; Langton, O'Malley, \& Bruce, 1996). This is consistent with the results of neurophysiological and neuroimaging studies that investigated the response properties of STS neurons (see Allison et al., 2000, for a review).

Other directional symbolic cues, such as arrows, might also produce a Simon effect (as was recently shown by Masaki, Takasawa, \& Yamazaki, 2000). In addition, there is preliminary evidence that arrow cues trigger automatic shifts of spatial attention (Tipples, 2002), as does gaze direction. These findings, however, do not call into question the existence of a specialized processing mechanism for eye gaze. In fact, the processing mechanism for eye gaze might be innate (see, e.g., Batki et al., 2000), whereas arrows and perhaps other social cues (e.g., hand gestures) no doubt acquire a directional value through experience. Our study is in accord with the notion that eye-like stimuli have a special status in visual processing and provide early constraints on the allocation of spatial attention.

\section{REFERENCES}

Allison, T., Puce, A., \& McCarthy, G. (2000). Social perception from visual cues: Role of the STS region. Trends in Cognitive Sciences, 4, 267-278.

Argyle, M., \& Cook, M. (1976). Gaze and mutual gaze. Cambridge: Cambridge University Press.

BARON-COHEN, S. (1994). How to build a baby that can read minds:
Cognitive mechanisms in mindreading. Current Psychology of Cognition, 13, 513-552.

Batki, A., Baron-Cohen, S., Wheelwright, S., Connellan, J., \& Ahluwalia, J. (2000). Is there an innate gaze module? Evidence from human neonates. Infant Behavior \& Development, 23, 223-229.

Bruce, C., Desimone, R. \& Gross, C. G. (1981). Visual properties of neurones in a polysensory area in the superior temporal sulcus of the macaque. Journal of Neurophysiology, 46, 369-384.

Butterworth, G., \& Jarrett, N. (1991). What minds have in common is space: Spatial mechanisms serving joint visual attention in infancy. British Journal of Developmental Psychology, 9, 55-72.

Corbetta, M., Shulman, G., Miezin, F., \& Peterson, S. (1995). Superior parietal cortex activation during spatial attention shifts and visual feature conjunction. Science, 270, 802-805.

Driver, J., Davis, G., Kidd, P., Maxwell, E., Ricciardelli, P., \& BARON-COHEN, S. (1999). Gaze perception triggers visuospatial orienting by adults in a reflexive manner. Visual Cognition, 6, 509-540.

Friesen, C. K., \& Kingstone, A. (1998). The eyes have it! Reflexive orienting is triggered by nonpredictive gaze. Psychonomic Bulletin \& Review, 5, 490-495.

HiEtanen, J. K. (1999). Does your gaze direction and head orientation shift my visual attention? NeuroReport, 10, 3443-3447.

Hoffman, E. A., \& Haxby, J. V. (2000). Distinct representations of eye gaze and identity in the distributed human neural system for face perception. Nature Neuroscience, 3, 80-84.

Hommel, B., Pratt, J., Colzato, L., \& Godisn, R. (2001). Symbolic control of visual attention. Psychological Science, 12, 360-365.

Hood, B. M., Willen, J. D., \& Driver, J. (1998). Adult's eyes trigger shifts of visual attention in human infants. Psychological Science, $\mathbf{9}$, 53-56.

Kingstone, A., Friesen, C. K., \& Gazzaniga, M. S. (2000). Reflexive joint attention depends on lateralized cortical connections. Psychological Science, 11, 159-166.

LANGTON, S. R. H. (2000). The mutual influence of gaze and head orientation in the analysis of social attention direction. Quarterly Journal of Experimental Psychology, 53A, 825-845.

Langton, S. R. H., \& BRUCE, V. (2000). You must see the point: Automatic processing of cues to the direction of social attention. Journal of Experimental Psychology: Human Perception \& Performance, 26, 747-757.

Langton, S. R. H., O’Malley, C., \& Bruce, V. (1996). Actions speak no louder than words: Symmetrical cross-modal interference effects in the processing of verbal and gestural information. Journal of Ex- 
perimental Psychology: Human Perception \& Performance, 22, 1357-1375.

Langton, S. R. H., Watt, R. J., \& Bruce, V. (2000). Do the eyes have it? Cues to the direction of social attention. Trends in Cognitive Sciences, 4, 50-59.

Lu, C. H., \& Proctor, R. W. (1995). The influence of irrelevant location information on performance: A review of the Simon and spatial Stroop effects. Psychonomic Bulletin \& Review, 2, 174-207.

Masaki, H., Takasawa, N., \& YamazaKi, K. (2000). An electrophysiological study of the locus of the interference effect in a stimulusresponse compatibility paradigm. Psychophysiology, 37, 464-472.

Maurer, D. (1985). Infant's perception of facedness. In T. Field \& M. Fox (Eds.), Social perception in infants (pp. 77-100). Norwood, NJ: Ablex.

Perrett, D. I., Smith, P. A. J., Potter, D. D., Mistlin, A. J., Head, A. S., Milner, A. D., \& Jeeves, M. A. (1985). Visual cells in the temporal cortex sensitive to face view and gaze direction. Proceedings of the Royal Society of London: Series B, 223, 293-317.

Posner, M. I. (1980). Orienting of attention. Quarterly Journal of Experimental Psychology, 32, 3-25.

Simon, J. R., \& CRAFT, J. L. (1970). Effects of an irrelevant auditory stimulus on visual choice reaction time. Journal of Experimental Psychology, 86, 272-274.

STERNBERG, S. (1969). The discovery of processing stages: Extensions of Donders's method. Acta Psychologica, 30, 276-315.
Tagliabue, M., Zorzi, M., Umiltà, C., \& Bassignani, F. (2000). The role of LTM links and STM links in the Simon effect. Journal of Experimental Psychology: Human Perception \& Performance, 26, 648670.

TipPles, J. (2002). Eye gaze is not unique: Automatic orienting in response to uninformative arrows. Psychonomic Bulletin \& Review, 9 , 314-318.

Umiltà, C., \& Nicoletti, R. (1990). Spatial S-R compatibility. In R. W. Proctor \& T. G. Reeve (Eds.), Stimulus-response compatibility: An integrated perspective (pp. 89-116). Amsterdam: North-Holland. Valenza, E., Simion, F., Macchi Cassia, V., \& Umiltà, C. (1996). Face preference at birth. Journal of Experimental Psychology: Human Perception \& Performance, 22, 892-903.

Vecera, S. P., \& Johnson, M. H. (1995). Gaze detection and the cortical processing of faces: Evidence from infants and adults. Visual Cognition, 2, 59-87.

Wicker, B., Michel, F., Henaff, M.-A., \& Decety, J. (1998). Brain regions involved in the perception of gaze: A PET study. NeuroImage, 8, 221-227.

Zorzi, M., \& Umiltà, C. (1995). A computational model of the Simon effect. Psychological Research, 58, 193-205.

(Manuscript received July 18, 2000; revision accepted for publication April 22, 2002.) 